\title{
Exploring Transferred Information for Daur Traditional Craftwork
}

\author{
Zhuo Bian ${ }^{1}$ and Liangliang Wang ${ }^{2}$ \\ ${ }^{1}$ Art Academy of Northeast Agriculture University, Harbin 150001, China; \\ ${ }^{2}$ State Key Laboratory of Robotics and System, Harbin 150001, China;

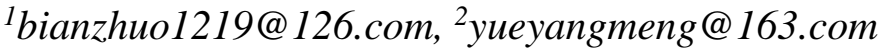

\begin{abstract}
This paper focuses on the disappearing Chinese Daur traditional craftwork on the graphical scale to reveal the great value of Daur culture from the culture conservation perspective. Specifically, typical Daur craftwork samples are surveyed and selected as the objective for analysis, based on which, color features and interest poins feature are respectively extracted employing our color card quantization method and Scale Invariant Feature Transform technique. Moreover, in order to apply the two extracted features into practice, principle component analysis scheme is utilized to tower the complexity and improve the accuracy of the feature information. experimental results are accomplilshed on the collected Daur craftwork dataset, which also proves the applicability and efficiency of our approach.
\end{abstract}

Keywords: Daur culture, Daur traditionaleraftwork, Color card quantization, Interest points extraction

\section{Introduction}

Daur nation is one of the fifty-six nations of China, mainly located in the Neimenggu and Heilongjiang province 11]. In spite of there are only twenty thousand people in Daur nation, it has a significant and excellent history which is dated back to Tang Dynasty [2]. Agriculture culture and traditional craftwork play a rather important part in Daur cluture, as a good instance, Daur utensil $[3]$ is very practical and popular, as well, has a big aesthetic value.

Nowadays, Daur nation and its culture are disappearing with the development of society. On the other hand, conservation of the Daur traditional culture and the precious historic craftwork are attracting more and more attention and have become a hot topic. Instead of directiy using conventional manual technique or archeology knowledge, our work aims at exploiting the transferred information revealed by graphical features of Daur traditional craftwork. Two graphical feature are extracted: color and interest points. Particularly, color card quantization method is proposed to analyze the color feature of samples collected via our survey. Furthermore, SIFT (Scale-Invariant Feature Transform) 4. technique is applied to represent interest points feature on the fundamental pixel scale. In order to get an acceptable comprehensive performance of the graphical feature, PCA (Principle Component Analysis) [5] strategy is employed to both reduce the complexity of representation descriptor and improve the accuracy of our approach. Experiments are carried out using typical samples from our Daur dataset, which proves that our system has good tolerance for accurate feature representation from different examples in even complicated scenarios and can be used for the future Daur culture conservation. 


\section{Color Samples Quantization}

In this section, we detail our work in two aspects: first, we introduce our dataset, based on this, we present our color extraction and analysis method.

\subsection{Historic Building Dataset}

In order to provide basic support for later scientific quantitative description, we build up our dataset using specific samples of brick hark which is most representative in Daur craftwork culture. All collected samples are set to be $320 \times 240$ size, and saved as png format. Examples from our dataset are shown in Figure 1.
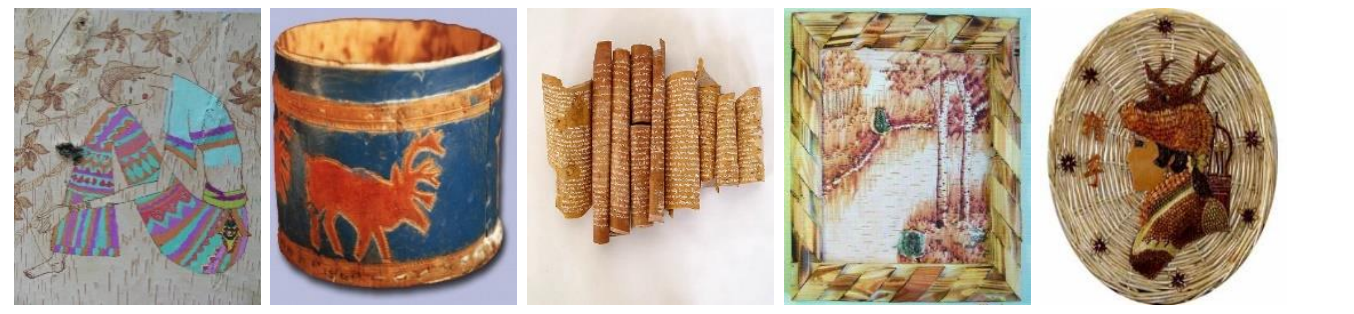

Figure 1. Example Samples of Our Daur Craftwork Dataset

As to making a brick hark craftwork, our survey-shows that at least two layers brick hark are needed for suture using animal's muscle both on the outer side, then, the outer layer of each brick hark is connected to craftwork's bottom via embedding a willow twig. Our dataset consists of different samples varied on background, color composition, and object formulation.

\subsection{Color Feature Representation}

To achieve color quantization for different samples, color histogram is applied in HSV space by statistics of the atisfied pixelk on the whole image.

HSV color space can be interpreted as a cone subset in the cylinder coordinate system. $\mathrm{V}$ ranges from 0 to 1 corresponding to respectively from the peak to the bottom of the cone, and $\mathrm{H}$ is decided by the angel rotated around the $\mathrm{V}$ direction while $\mathrm{S}$ indicates the radius of the cone plane. In our algorithm, the locations of image pixels are not recorded, and the distribution of parameters H, S and V are analyzed, so that HSV space is divided to many subspaces each of which is distinguished by pixels with similar features.

\section{Interest Point Analysis}

\subsection{Interest Point Extraction}

Initially, SIFT interest points are extracted to represent image local features on several sales which avoiding noises caused by image rotation, illumination variance and others. S.FT algorithm can be divided into 3 steps:

(1) Extrama detection on space scales. Let $I(x, y)$ denotes an image in the brightness intensity level. First, use Gaussian convolution kernel to smooth the image, and the space scales are defined as different layers using different Gaussian variance kernel, after which image sampling process is carried out, the space scale is:

Where:

$$
L(x, y, \sigma)=G(x, y, \sigma) * I(x, y)
$$

$$
G(x, y, \sigma)=\frac{1}{2 \pi \sigma^{2}} e^{-\left(x^{2}+y^{2}\right) / 2 \sigma^{2}}
$$

$\sigma$ denotes the stand deviation of Gaussian distribution, which also determines the scale space of the image. After that, the space is further computed using the following equation: 


$$
D(x, y, \sigma)=(G(x, y, k \sigma)-G(x, y, \sigma)) * I(x, y)=L(x, y, \mathrm{k} \sigma)-L(x, y, \sigma)
$$

(2) Key points localization. By conic curve fitting, real extrema can be found based on the scale space fitting function:

Where $\mathrm{X}=(\mathrm{x}, \mathrm{y}, \sigma)^{T}$.

$$
D(X)=\mathrm{D}+\frac{\partial D^{T}}{\partial X} X+\frac{1}{2} X^{T} \frac{\partial^{2} D}{\partial X^{2}} X
$$

(3) Deciding the direction of interest points. Based on the local gradient of the image, give each point a direction.

(4) Interest point description: Calculate the gradient infromation in a interest point centered window, by which the description can be achieved by statistics.

\subsection{PCA of Interest Point}

As the SIFT descriptors can be expressed using a matrix $\mathrm{X}$, in order to reduce the redundancy of the extracted interest points information, as well, to improve the accuracy of the interest points descriptors, PCA is applied which can be summarized as 3 stages:

(1) Compute the covariance matrix $S$ of the initial data matrix $X$.

(2) Calculate the eigenvectors of matrix S, and order these eigenvectors from large to small.

(3) Project the matrix $X$ to the space which eigenvectors deternine then to replace the matrix X using principle components.

After PCA processing, we can use the new principle components according to the contribution of the new vectors.

\section{Experimental Results}

In our experiments, initially, Gpical samples from our Daur craftwork dataset are selected. Based on this, graphical features mncluding color and interest point are respectively extracted for analysis on micro scale of craftwork.

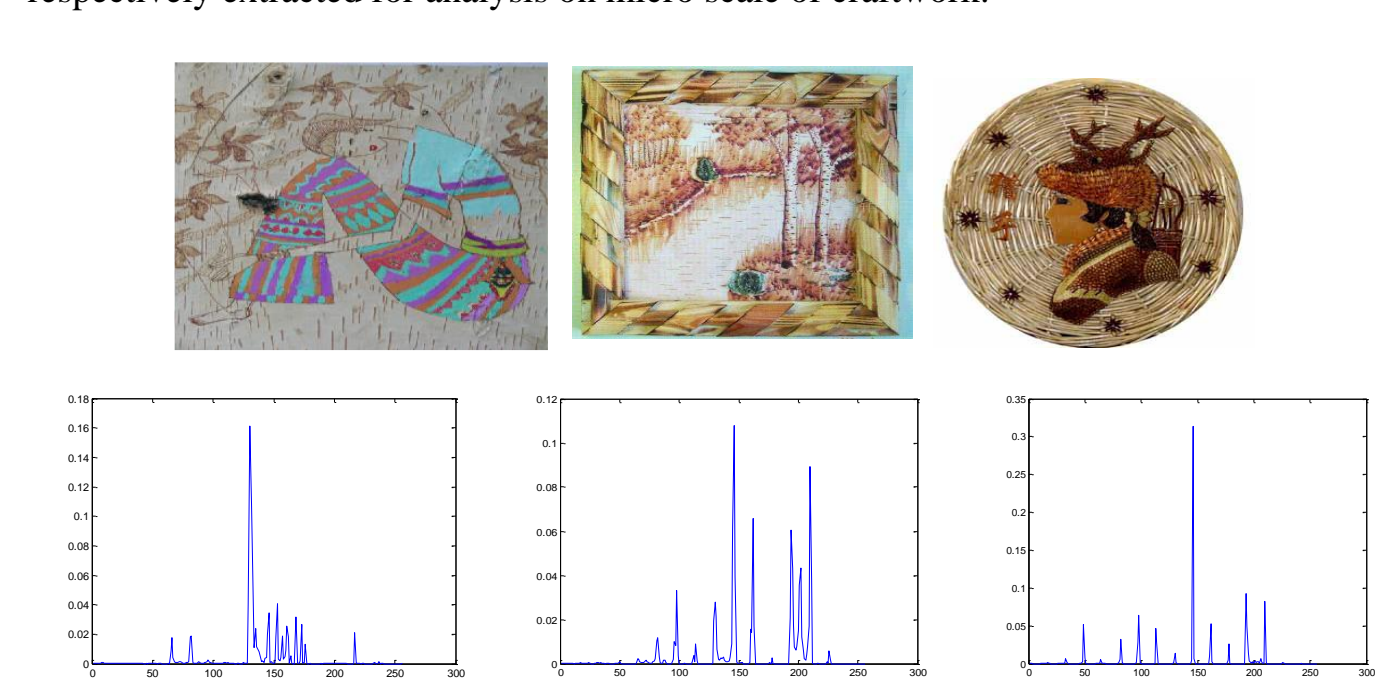

\section{Figure 2. Example Results of Color Histogram: Images in the First Column are from Our Dataset and Images in the Second Column are the Corresponding Color Histogram}

Figure 2 shows some example results of our color histogram scheme. Parameters of $\mathrm{H}$, $\mathrm{S}$ and $\mathrm{V}$ are set as: $16,4,4$, so that the histogram ranges from 0 to 256 as shown in the horizontal direction, and in the vertical direction of histogram, the value ranges from 0 to 1 as the quantization of the method. 

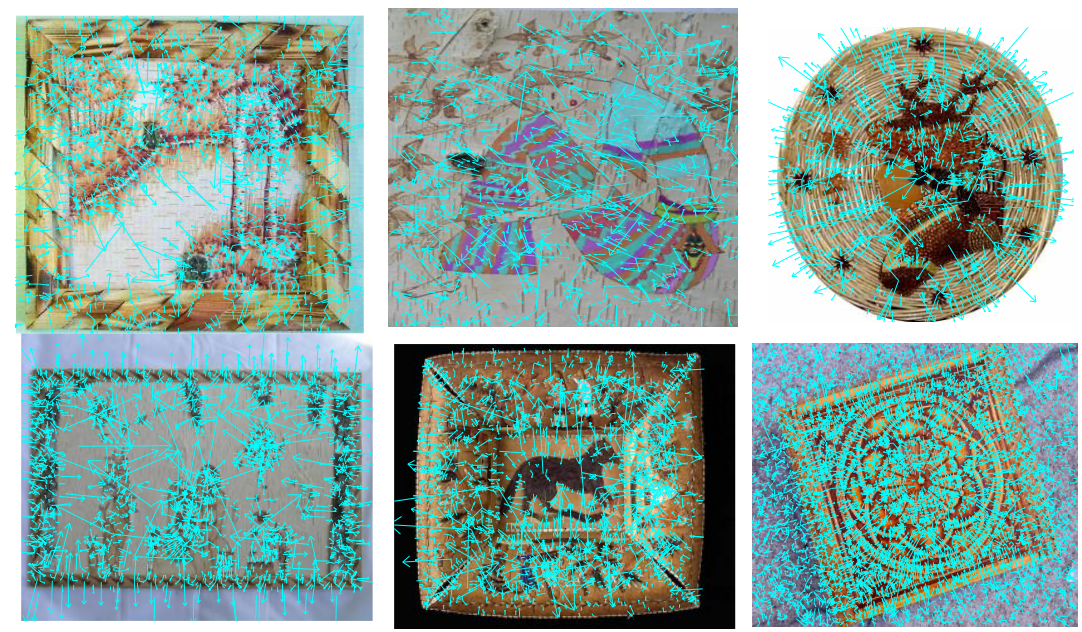

\section{Figure 3 Example Results of Interest Points Extracted on Daur Craftwork Samples Using SIFT.}

Figure 3 illustrates the interest points extraction based on SIFT algorithm using Daur craftwork examples. The scale extrema window is set as $4 \times 4$, and the standard deviation $\sigma$ is set as 1.6. From Figure 3, we can infer that SIFT points are very suited for analysis of Daur craftwork as the image can be clearly represented whilemerits on graphical level are guaranteed.

Finally, PCA is executed on SIFT descriptors $90 \%$, contribution rate is set in our experiment and the new projected data are stored for future application.

\section{Conclusion}

This paper presents a graphical fealure representation system for Daur craftwork culture. A craftwork sample dataset is first built up, based on which, color and interest point feature are respectively extracted based on color histogram and SIFT descriptors to analyze the micro characteristies of these samples. Experiments validate our algorithm which also prove that our system is of great value in the future conservation or analysis for Daur ceaftwork culture. $4 t$ is easy to imagine that our graphical system is suited for image analysis and feature extraction in many craftwork culture related conservation work. In our future york, we are very interested to construct a larger Daur craftwork samples dataset and combine more graphical descripotrs to analyze the inline features of this conventional culture, besides, more conventional culture especially in Heilongjiang province will be discussed using computer graphical methods for conservation purpose.

\section{References}

11 - Lu, "Daur Traditional Handicraft Research", Neimenggu Agricultural University Thesis, (2011), pp. 3 -6.

[2] Hualinge, "Daur Soren Source Survey", Qing Dynasty Publisher, Beijing, (1833), pp. 22-25.

[3] X. Guo and X. Er, "Daur Relics Catalog", Neimenggu Publisher, Huhehaote, (2008), pp. 16-17.

[4] D. Lowe, "Distinctive image features from scale-invariant keypoints", Int. J. Comput. Vis., vol. 60, (2004), pp. 91-110.

[5] S. T. Lim and D. F. W. Yap, "Medical image compression using block-based PCA algorithm", 2014 International conference on Computer, Communications, and Control Technology, (2014), pp. 171-175. 


\section{Author}

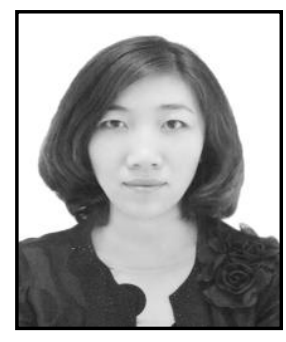

Zhuo Bian, received MS Of Design Management in 2006 at Harbin Institute of Technology. Now she is a lecture in Art Academy of Northeast Agriculture University, Harbin, CHN. Her current research interest includes visual communication design and humancomputer interaction design.

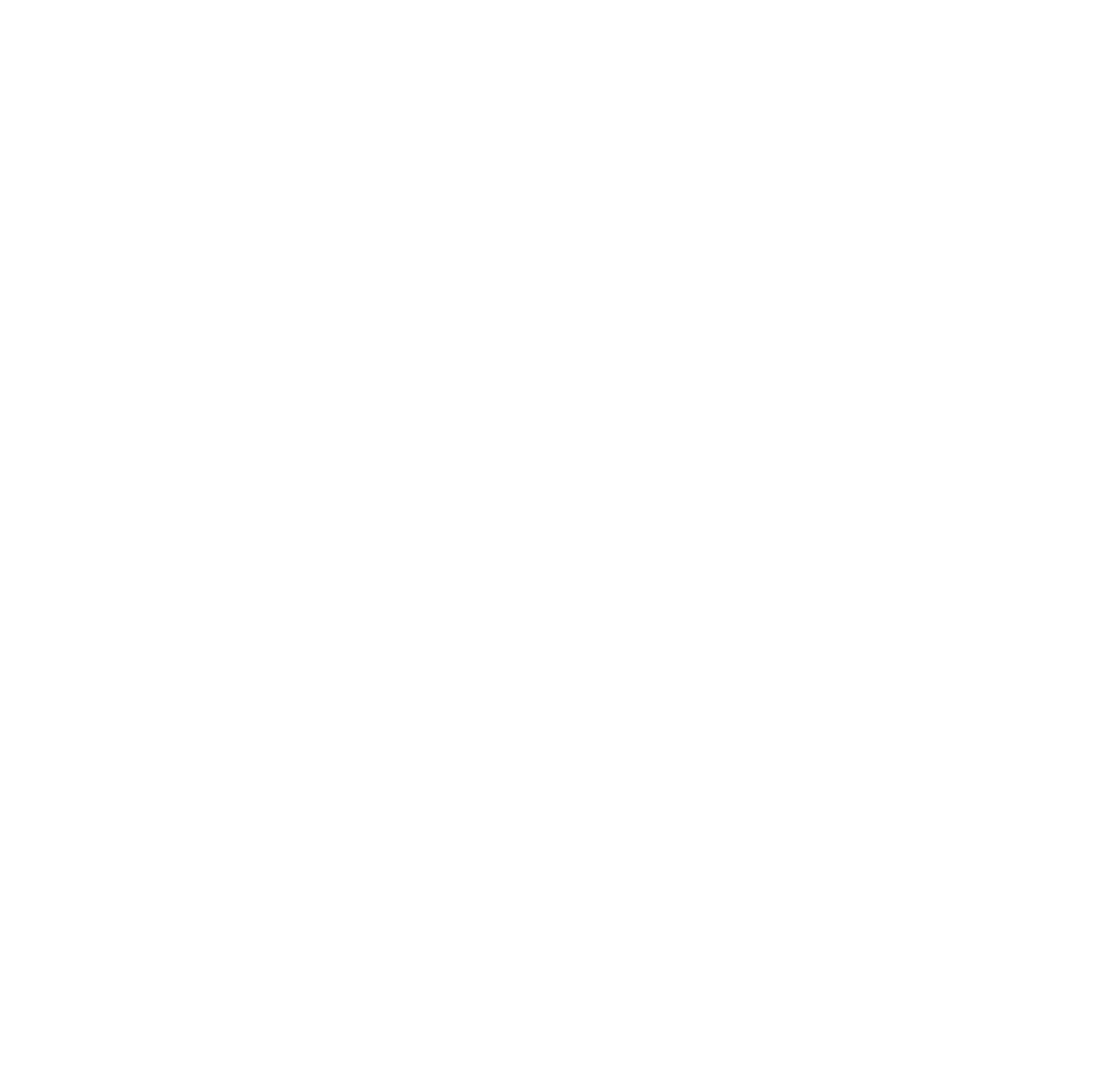


International Journal of Multimedia and Ubiquitous Engineering

Vol.11, No.11 (2016)

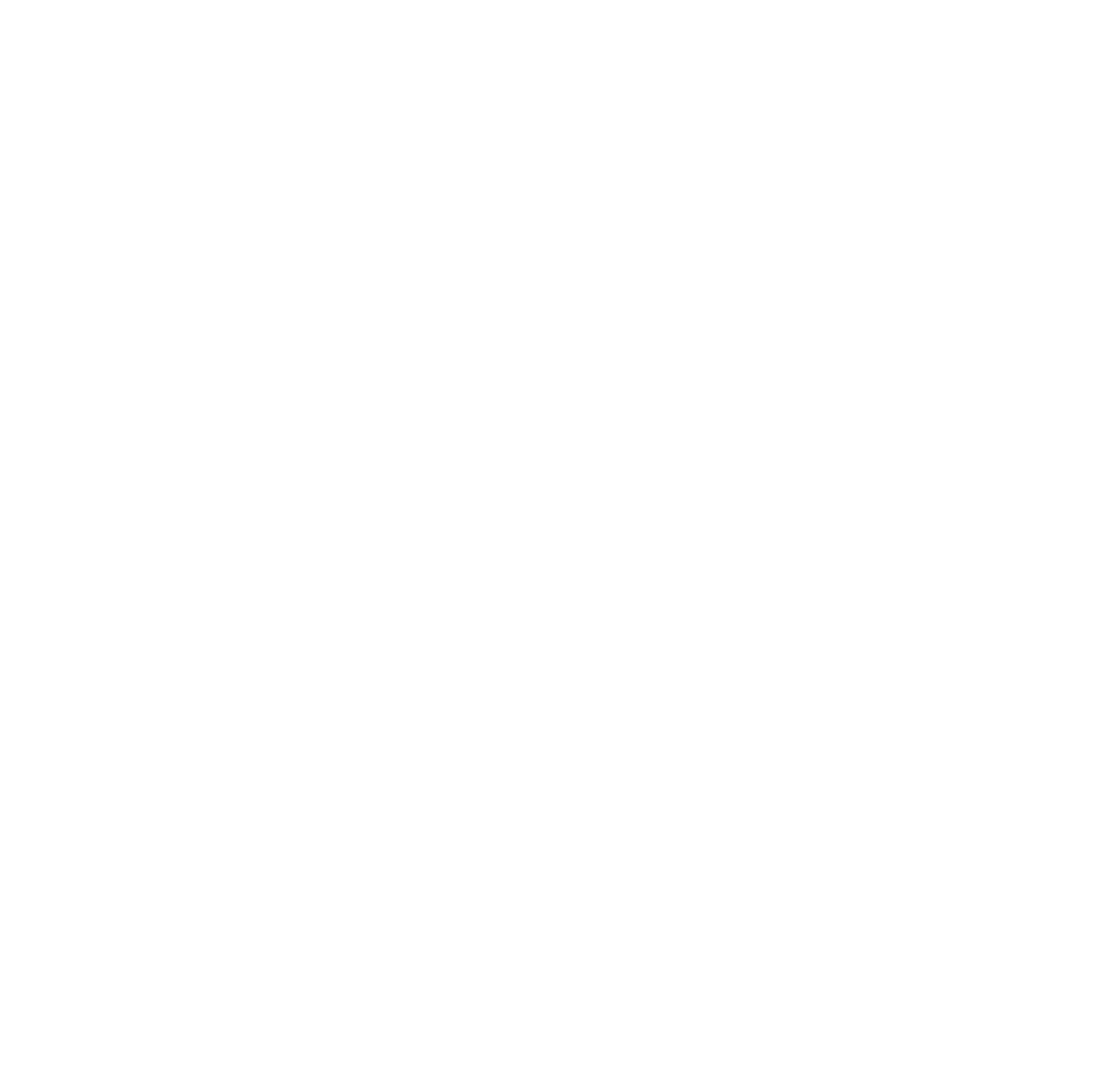

